\title{
Time-varying noise control in motorcycle helmets
}

\author{
Demián García Violini ${ }^{1,2, *}$, Ricardo S. Sánchez Peña ${ }^{1,3, \dagger}$ and Ariel Velis ${ }^{4, \ddagger}$ \\ ${ }^{1}$ Buenos Aires Institute of Technology (Instituto Tecnológico de Buenos Aires), Av. Madero 399, CABA, Argentina \\ ${ }^{2}$ National University of Quilmes, Roque Sáenz Peña 352, Bernal, Buenos Aires, Argentina \\ ${ }^{3}$ National Council of Technical and Scientific Research (CONICET), Av. Rivadavia 1917, CABA, Argentina \\ ${ }^{4}$ Acoustics and Lighting Laboratory (LAL), Cno. Centenario 505-508, M. B. Gonnet., Buenos Aires, Argentina
}

(Received 25 July 2014, Accepted for publication 28 August 2014)

Keywords: Motorcycle helmets, Active noise control, $\mathscr{H}_{\infty}$ optimal control, Linear parameter-varying control PACS number: 43.50.Ki, 43.50.Hg, 43.50.Lj [doi:10.1250/ast.36.333]

\section{Introduction}

Motorcyclists are usually exposed to high noise levels that increase with speed. There have been a variety of medical studies that confirm that the prolonged exposure to excessively noisy environments may cause health problems, such as, noise-induced hearing loss (NIHL) [1-4]. Active noise control (ANC) has an extensive reference bibliography [5,6], but there have been very few ANC works on motorcycle helmets [1,7-9], probably due to the time-varying nature of their dynamics.

Here, the active noise control problem in motorcycle helmets is studied, which has an intrinsic time-varying behavior. To achieve a reasonable noise attenuation, the noise source and its dynamic characteristics are computed through an experimental identification process. Also, in a robust control framework [10,11], the time-varying dynamics can be either considered as a dynamic uncertainty or treated as it is. Here a time-varying feedback (FB) controller is proposed and compared with a robust linear time-invariant (LTI) controller. The LTI controller optimizes against the lack of knowledge of the model dynamics using $\mathscr{H}_{\infty}$ optimal control.

The time-varying approach is designed to have an attenuation level that increases with the speed between the helmet and the air (since the noise level increases with speed) using a linear parameter-varying (LPV) controller. The main purpose here is to take advantage of this time-varying controller to tune the attenuation level in accordance with the noise environment. In this way, the attenuation is higher at speeds where the external noise is louder and lower at reduced velocities. Such tuning cannot be performed with LTI controllers, which provide a constant attenuation at all speeds. The result is illustrated by an experimental simulation, which uses the noise obtained from a freeway at different speeds.

\section{Results}

\subsection{Experimental setup}

Three different experiments were performed: an experiment on the noise dynamics, identification tests, and an experiment on controller implementation. The first involved measuring the sound pressure level simultaneously with the

\footnotetext{
*e-mail: ddgarcia@itba.edu.ar

†e-mail: rsanchez@itba.edu.ar

‡e-mail: arielvelis@yahoo.com
}

relative speed between the air and the helmet. This setup was placed on a car driven at different speeds. In the identification tests, the voltage on the headphones and the pressure in the ear canal were measured.

The following hardware was used in all the experiments: a pair of Sennheiser HD 280 headphones placed inside the helmet, a Testo 06280036 anemometer for the car speed measurement, a dummy head specifically built for this purpose (Fig. 1), an anti-vibration platform to mount the dummy on the car, a Knowles BT-21759 microphone placed at the ear canal of the dummy and a National Instruments (NI) CompactRIO system (cRIO 9075, with 9205 and 9263 modules for analog input and output, respectively) for the data acquisition and real-time control. The control was implemented on a Xilinx FPGA in the CompactRIO. A complete view of the experimental setup is shown in Figs. 1 and 2 .

\subsection{Noise dynamics and identification}

Concerning the noise characterization, Fig. 3 shows the power spectral density (PSD) for different velocities.

The identification tests were carried out using six sinusoidal sweeps as system excitation signals. Between each sweep, the system was perturbed (by changing the helmet's position, moving the error microphone and headphone positions, etc.) to include various practical situations. From the tests, a nominal model $G_{0}(s)$ and an uncertainty weight were obtained using subspace methods [12,13], which were used to design robust controllers. Figure 4 illustrates how the nominal model (in black) has a good data fit with the experimental transfer functions (in red) for all the tests.

\subsection{Controller design and implementation}

An $\mathscr{H}_{\infty}$ controller was designed to ensure the maximum attenuation level and robust stability for all relative velocities between the air and the helmet. A fixed performance weight $W_{p}(s)=\frac{7540}{s+2 \pi 300}$ was designed to increase performance at lower frequencies. For practical reasons, to produce a controller that does not require a high sampling rate, a linear matrix inequality (LMI) region [14] was added so that a cutoff frequency of $25 \mathrm{kHz}$ was achieved in the closed loop.

The LPV controller was designed so that the resulting attenuation could be modulated by the speed in accordance with a desired profile, for example, when the environmental noise is low, the controller can be turned off. To achieve this 


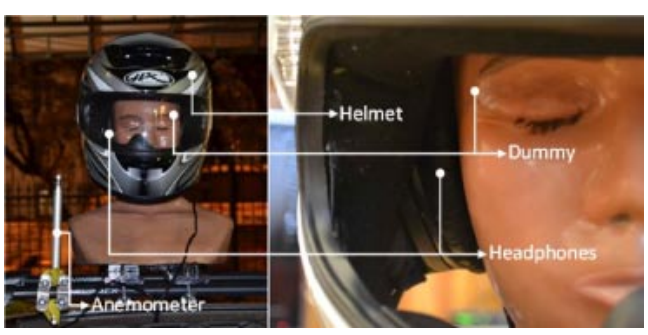

Fig. 1 Experimental setup on the car showing head, anemometer, helmet, and headphone.

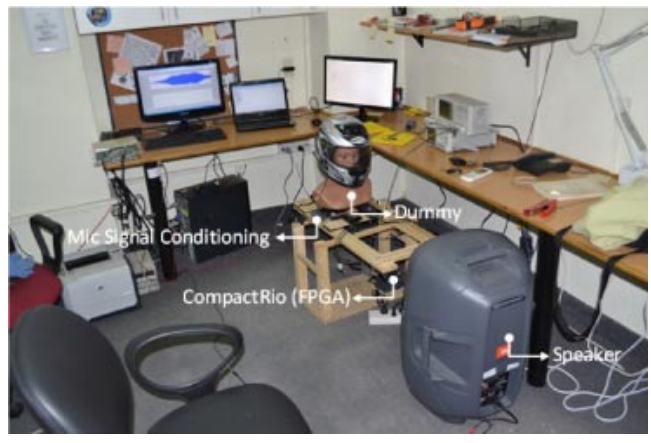

Fig. 2 Identification and control experimental setup.

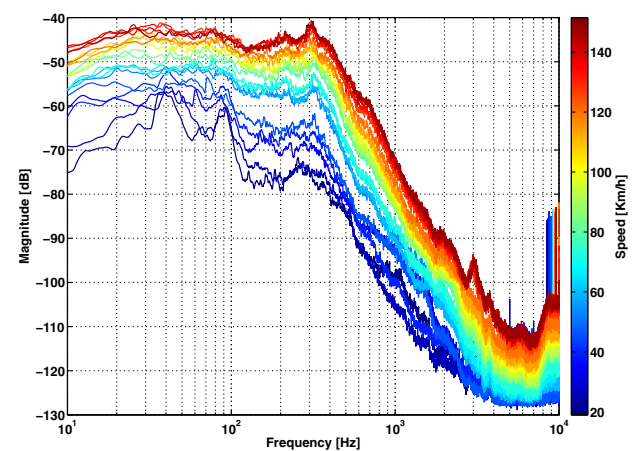

Fig. 3 Estimated PSD (Thomson multitaper method) for the range of air velocities of $20-140 \mathrm{~km} / \mathrm{h}$.

objective, a speed-varying performance weight is proposed as follows:

$$
\begin{aligned}
W_{p}(s, v)= & {\left[\begin{array}{cc}
-A_{w}(v) & 1 \\
B_{w}(v) & 0
\end{array}\right] } \\
= & {\left[\begin{array}{cc}
-f_{1}+\frac{f_{2}-f_{1}}{v_{2}-v 1} v_{1} & 1 \\
k_{1}-\frac{k_{2}-k_{1}}{v_{2}-v 1} v_{1} & 0
\end{array}\right]+\left[\begin{array}{cc}
-\frac{f_{2}-f_{1}}{v_{2}-v 1} & 0 \\
\frac{k_{2}-k_{1}}{v_{2}-v 1} & 0
\end{array}\right] v, }
\end{aligned}
$$

where the notation $\left[\begin{array}{cc}A & B \\ C & D\end{array}\right]$ refers to the $(A, B, C, D)$ state-space matrices. Here the LPV system is a polytopic model with the real-time measured speed as the varying parameter $v \in$ $\left[v_{1}, v_{2}\right]=[0,140] \mathrm{Km} / \mathrm{h}$ with $k_{1}=12,566.37, k_{2}=7,539.82$, $f_{1}=10^{4}$, and $f_{2}=300$.

Figure 5 shows the LPV system attenuation level as a function of speed. Note that the LPV system shows gradual attenuation depending on the relative speed measured by the
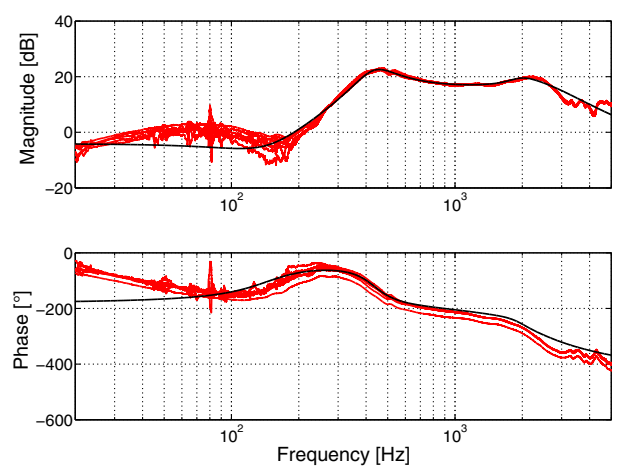

Fig. 4 Experimental transfer functions (red) and identified nominal model $G_{0}(\jmath \omega)$ (black).

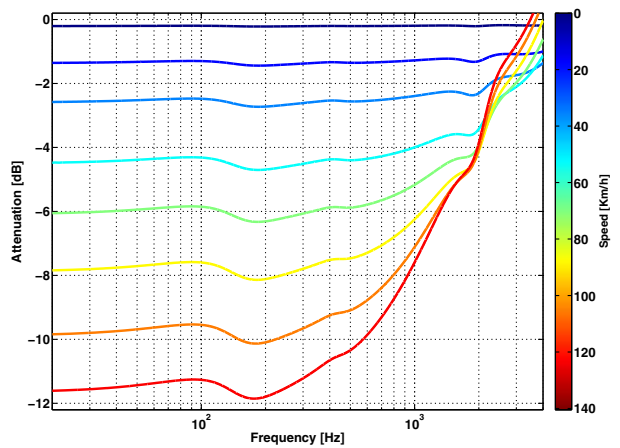

Fig. 5 Attenuation level at each speed.

anemometer. The $\mathscr{H}_{\infty}$ controller sensitivity function matches that of the LPV system at the maximum motorcycle velocity (approx. $140 \mathrm{~km} / \mathrm{h}$, lower red curve in Fig. 5), because at this speed it provides the best attenuation with robust stability guaranteed.

The controller was implemented on a Xilinx FPGA contained in an NI CompactRIO cRIO-9075 system. The discretization of the controller was carried out through a Tustin transform with a $50 \mathrm{kHz}$ sampling rate. In terms of practical issues, two aspects were taken into account: the controller implementation structure (balanced state-space realization) and fixed-point quantification.

The controller programmed in the FPGA was tested using the data acquired at the noise characterization stage (Sect. 2.2) using a JBL Eon 315 flat response speaker, as shown in Fig. 2. The velocity measurement was performed using an additional analog input that was sensed by the anemometer. To carry out this experiment, an auxiliary system based on an NI PCIe-6323 data acquisition card was used to write the signals (waveform or speed profiles) to each recipient (speaker and CompactRIO). In this way, the control hardware (FPGA) was not overtaxed.

Figure 6 shows the results of both controllers as compared with the open-loop response for a particular speed profile. The attenuation levels are indicated in Table 1. The LPV attenuations were computed at $655 \mathrm{~ms}$ intervals by calculating the average speed and attenuation. Notice that the $\mathscr{H}_{\infty}$ controller achieves a constant attenuation level regardless of 



Fig. 6 Blue: open-loop signal. Red: output signal of LPV closed loop. Green: output signal of $\mathscr{H}_{\infty}$ closed loop. Upper plot: output signal level. Lower plot: speed profile.

Table 1 Controller attenuation levels at different speeds.

\begin{tabular}{ccc}
\hline $\begin{array}{c}\text { Speed } \\
(\mathrm{km} / \mathrm{h})\end{array}$ & $\begin{array}{c}\mathscr{H}_{\infty} \\
(\mathrm{dB})\end{array}$ & $\begin{array}{c}\text { LPV } \\
(\mathrm{dB})\end{array}$ \\
\hline 59.5 & 10.85 & 2.2 \\
64 & 11.73 & 2.63 \\
73.6 & 11.39 & 2.39 \\
82.1 & 11.13 & 3.25 \\
96.2 & 11.66 & 4.52 \\
101.2 & 11.72 & 4.77 \\
113.2 & 11.59 & 6.83 \\
121.6 & 11.72 & 9.11 \\
127.8 & 12.03 & 12.32 \\
\hline
\end{tabular}

the air speed due to the fact that its dynamics are timeinvariant. In contrast, the LPV controller increases the attenuation level up to the maximum speed in accordance with its design. The output signal level with the LPV controller is similar to that for the $\mathscr{H}_{\infty}$ controller at the maximum speed, at which both were designed to have the same attenuation. Of course, different attenuation $v s$ speed functions can be achieved by modifying the design weight $W_{p}(s, v)$ in the LPV controller synthesis stage.

Regardless of the attenuation results, the alternative of having a controller that changes its performance as a function of the helmet speed is attractive. In this case, we have used the controller as a way of modulating the attenuation in accordance with the changes in the noise level vs velocity. In other cases, this time-varying controller can be applied to fit a particular performance $v s$ speed specification. For example, when the environmental noise is low, say below $40 \mathrm{~km} / \mathrm{h}$, the controller could be turned off.

\section{Conclusions and future research}

The potential of a time-varying LPV controller that can tune its noise attenuation in accordance with the velocity is a prominent feature of this work. This controller has also been compared with a time-invariant version ( $\mathscr{H}_{\infty}$ controller), which produces good results in the case that a simpler robust controller is required. In the near future, a combination of the LPV controller with a feedforward controller will be pursued to further reduce the noise levels.

\section{References}

[1] L. Liu, S. M. Kuo and K. P. Raghuathan, "Active noise control for motocycle helmets," Int. J. Inf. Commun. Eng., 6, 102-107 (2010).

[2] B. Kirchner, E. Evenson, R. Dobie, P. Rabinowitz, R. K. J. Crawford and W. Hudson, "Occupational noise-induced hearing loss," ACOEM Guid. Statement, 54(1), (2012).

[3] C. Jordan, "Noise induced hearing loss in occupational motorcyclists," J. Envirom. Health Res., 3, 373-382 (2004).

[4] W. V. Moorhem, K. Sheperd, T. Magleby and G. Torian, "The effects of motorcycle helmets on hearing and the detection of warning signals," J. Sound Vib., 8, 39-49 (1981).

[5] P. Nelson and S. Elliot, Active Control of Sound (Academic Press, 1992).

[6] S. Kuo and D. Morgan, Active Noise Control Systems: Algorithms and DSP Implementations (John Wiley \& Sons, New York, 1995).

[7] M. Lower, D. Hurst and A. Thomas, "Noise levels and noise reduction under motorcycle helmets," Proc. Inst. Acoust. (1994).

[8] R. Castañe-Selga and R. S. Sánchez Peña, "Active noise hybrid time-varying control for motocycle helmets," IEEE Trans. Control Syst. Technol., 18, 602-612 (2010).

[9] C. H. Brown and M. S. Gordon, "Motorcycle helmet noise and active noise reduction," Open Acoust. J., No. 4, pp. 14-24 (2011).

[10] K. Zhou, J. C. Doyle and K. Glover, Robust and Optimal Control (Prentice Hall, 1996).

[11] R. S. Sánchez Peña and M. Sznaier, Robust Systems Theory and Applications (John Wiley \& Sons, 1998).

[12] P. Van Overschee and B. De Moor, "N4SID: subspace algorithms for the identification of combined deterministic and stochastic systems," Automatica, 30, 75-93 (1994).

[13] M. Verhaegen, "Identification of the deterministic part of MIMO state space models given in innovations form from inputoutput data," Automatica, 30, 61-74 (1994).

[14] M. Chilali and P. Gahinet, " $\mathscr{H}_{\infty}$ control design with pole placement constraints: An LMI approach," IEEE Trans. Autom. Control, 41, 358-367 (1996). 